\title{
Desempeños efectivos de alumnos de educación primaria
}

\author{
Esteban García Hernández \\ Heurística Educativa \\ estebangarcia_h@yahoo.com
}

\begin{abstract}
Resumen
En este trabajo se presenta uno de los instrumentos diseñados para realizar el monitoreo de la propuesta del Consejo Nacional de Fomento Educativo, denominada Dialogar y Descubrir, que es considerada como central para brindar educación primaria a niños de 6 a 14 años que habitan en comunidades rurales, indígenas o campamentos agrícolas. El instrumento de investigación se conforma por una serie de 12 actividades que se construyeron para rescatar información en relación a los conocimientos de los alumnos que cursan su educación primaria en Centros Comunitarios, además del tipo de tareas social y culturalmente significativas que son capaces de realizar. Los datos que se recaban de la aplicación del instrumento permiten un análisis de tipo inductivo del que se deriva la identificación de diferentes niveles de desempeño de los alumnos, además en el marco del monitoreo brindan elementos para la explicitación de prácticas pedagógicas que se desarrollan por instructores comunitarios que reciben capacitación para aplicar la propuesta denominada Dialogar y Descubrir.
\end{abstract}

\section{Palabras clave}

Educación básica, monitoreo, aprendizaje, desempeños efectivos.

\section{Contexto}

El Consejo Nacional de Fomento Educativo (CONAFE) ofrece el servicio de primaria comunitaria a niños de 6 a 14 años, en localidades con un máximo de 29 niños con este rango de edades, además de niños migrantes que residen en campamentos agrícolas o albergues. La organización de las primarias comunitarias es multinivel: Nivel I. Equivale a primero y segundo grados en el sistema regular, Nivel II. Equivale a tercero y cuarto grados en el sistema regular y Nivel III. Equivale a quinto y sexto grados en el sistema regular.

Los alumnos son atendidos por instructores comunitarios que son capacitados para trabajar una propuesta educativa, con el apoyo de los libros de una serie denominada Dialogar y Descubrir. Dicha propuesta integra los contenidos curriculares del Plan y programas de estudio de la Secretaría de Educación Pública.

Durante el Ciclo escolar 2008-2009, Heurística Educativa, equipo de investigación y consultoría en educación radicado en la ciudad de Chihuahua, llevó a cabo el monitoreo de Dialogar y Descubrir, en una muestra de 16 Centros Comunitarios ubicados en 13 entidades federativas. Uno de sus objetivos 
generales del monitoreo fue la valoración de los recursos del modelo a partir de diversos elementos, tales como los aprendizajes efectivos de los estudiantes.

\section{Características y descripción del instrumento}

La base fundamental para valorar el modelo Dialogar y Descubrir, así como para identificar las estrategias más adecuadas para su enriquecimiento, fue el aprovechamiento académico de los alumnos. En muchos de los casos únicamente se contaba con resultados de pruebas estandarizadas (ENLACE).

En referencia al aprendizaje de los alumnos, el interés se focalizó en identificar información que permitiera conocer dos aspectos: lo que ellos saben y el tipo de tareas social y culturalmente significativas que son capaces de realizar.

Para lograr lo anterior se diseñó un instrumento para realizar una valoración de desempeños efectivos de alumnos que asisten a Centros Comunitarios CONAFE, con este instrumento se buscaba observar el desempeño de los alumnos, con la presentación de tareas o situaciones que les permitían demostrar de manera individual, su comprensión de un concepto o procedimiento en situaciones prácticas, a partir de la construcción de una respuesta, la realización de una acción o la creación de un producto. Shagoury y Miller (2000, p. 68) plantean que las producciones de los alumnos son fuentes importantes para entender procesos escolares y presentan una serie de técnicas, instrumentos y procedimientos que se pueden realizar en las aulas, incluso por los docentes que se interesan en asumir prácticas de investigación.

El instrumento se conformó por un total de 12 actividades, las dos primeras se subdividieron dos, por lo que se valoran un total de 14 desempeños de los alumnos a quienes se aplicó. Los fundamentos para el diseño de las actividades fueron los siguientes:

- Permiten obtener evidencias de aprendizajes y habilidades de los alumnos.

- No requieren el dominio de conceptos o procedimientos convencionales.

- Consideran contenidos programáticos y forman parte de acciones didácticas sugeridas tanto en el Plan y programas de estudio 1993 como en los manuales para el Instructor Comunitario de Dialogar y Descubrir.

- Es posible ubicarlas en una escala que permita observar niveles de logro.

Las actividades específicas que se diseñaron, presentan además las siguientes características:

- Hacen referencia a la comunidad inmediata de los alumnos; la totalidad de las actividades parten del contexto del niño y los saberes que pudiera tener de éste, incluso permiten realizar consultas sencillas con los adultos de la comunidad.

- Permiten además obtener información de la comunidad; los datos que los alumnos reportan posibilitan contar con datos adicionales de las actividades que en la comunidad se realizan, las formas de organización que se presentan, los problemas que existen y los cambios que se han observado de manera reciente, con esta información es posible complementar reportes de caso o triangular información.

- En general actividades propuestas son las mismas para los alumnos de los Niveles I, II y III; esto tienen la intención de establecer contrastes para identificar diferencias cualitativas en el desempeño de los alumnos.

- Los productos que se busca que 
los alumnos generen a partir de las actividades son:

Dibujos

Croquis

Ubicación de puntos cardinales en un plano.

Representaciones gráficas

Expresión oral (descripción con base en un dibujo y descripción y narración acerca de temas o procedimientos determinados).

Expresión escrita (registro de datos específicos, escritura con base en un organizador y con base en cuestionarios cortos).

A continuación se presentan las actividades que los evaluadores aplicaron.

Actividad 1A. Así es mi comunidad. Actividades del evaluador:

Pedir al alumno que realice un dibujo de su comunidad en el que identifique elementos naturales (montañas, valles, ríos, lagunas, sierras), casas, caminos y los principales lugares que la conforman y anote su nombre. Con base en el dibujo que presente el alumno, solicitarle que describa las características de los lugares señalados y las actividades que en ellos se realizan.

Actividad 1B. Así es mi comunidad. Actividades del evaluador:

Indicar al alumno que identifique los puntos cardinales en un espacio abierto y posteriormente pedirle que los señale en el dibujo de su comunidad.

Actividad 2A. ¿En dónde se localiza mi comunidad?

Actividades del evaluador:

Solicitar al alumno que elabore un croquis en el cual se ubique su comunidad, las comunidades aledañas y el camino para llegar a la cabecera municipal.
Actividad 2B. ¿En dónde se localiza mi comunidad?

Actividades del evaluador:

Preguntar al alumno acerca de las características específicas del croquis que realizó: lugares identificados caminos accidentes geográficos símbolos utilizados.

Actividad 3. Las plantas y los animales de la comunidad.

Actividades del evaluador:

Pedir al alumno que mencione el nombre de las plantas y animales de su comunidad y algunas de sus características. Solicitar al alumno que observe una planta de su comunidad, la dibuje y anote los siguientes datos: Nombre, lugar donde crece, descripción (color, forma, tamaño) y usos que tiene.

Actividad 4. ¿Quiénes formamos la comunidad?

Actividades del evaluador:

Pedir al alumno que haga un dibujo de su familia. Cuestionar al alumno acerca de las características de su familia y las familias de la comunidad con las que se relaciona (tipos de familia, integrantes, parentesco, actividades que realizan).

Actividad 5. ¿Cómo nos comunicamos en nuestra comunidad?

Actividades del evaluador:

Solicitar al alumno que observe en su comunidad portadores de texto (material escrito) y lo lleve a la escuela (puede ser en físico o una copia de éste). Pedir al alumno que lea el material colectado y comente: ¿Qué dice el texto?, ¿dónde estaba escrito?, ¿a quiénes va dirigido el mensaje?, ¿qué se pretende con dicho mensaje?

Actividad 6. ¿Qué actividades realizamos en la comunidad? 
Actividades del evaluador:

Dialogar con el alumno acerca de los oficios y trabajos que se realizan los habitantes de su comunidad, cómo los llevan a cabo y la importancia de éstos. Pedir al alumno que escriba la información en un cuadro como el siguiente:

¿Qué oficios o trabajos se realizan en tu comunidad?

¿En qué consisten?

¿Por qué son importantes?

Actividad 7. ¿Cómo compramos y cómo vendemos?

Actividades del evaluador:

Pedir al alumno que describa una situación de una transacción comercial (actividad de compra, venta o intercambio) en la que haya participado de manera reciente. ¿Qué compraste o qué vendiste? Describe cómo le hiciste para saber ¿cuánto cobrar o cuánto pagar? Anota las cuentas que hiciste.

Preguntar al alumno del Nivel I: ¿Qué puedes comprar con una moneda de 10 pesos? Describe con detalle ¿cómo lo sabes?, ¿cuánto dinero te sobraría? Solicitar al alumno que escriba su procedimiento en papel y explique las acciones que realizó.

Preguntar al alumno del Nivel II: ¿Qué puedes comprar con un billete de 20 pesos? Describe en detalle ¿cómo lo sabes?, ¿cuánto dinero te sobraría? Solicitar al alumno que escriba su procedimiento en papel y explique las acciones que realizó.

Preguntar al alumno del Nivel III: ¿Qué puedes comprar con un billete de 50 pesos? Describe en detalle ¿cómo lo sabes?, ¿cuánto dinero te sobraría? Solicitar al alumno que escriba su procedimiento en papel y explique las acciones que realizó.
Actividad 8. Nuestros juegos diarios. Actividades del evaluador:

Solicitar al alumno que describa juegos en los que participa en los que tenga que realizar cálculos. Describa los cálculos que realiza en los juegos y anote ejemplos de estos.

Actividad 9. ¿Cómo contamos y cómo calculamos

Actividades del evaluador:

Cuestionar a los alumnos acerca de cómo miden (se le puede preguntar de manera secuenciada por la medición de longitudes, áreas, volúmenes y tiempos), los habitantes de su comunidad (se puede decir que, al construir, preparar mezcla, preparar alimentos, trazar terrenos, sembrar, alimentar animales). En caso de que el alumno no lo haya mencionado se le puede preguntar directamente por las unidades de medida que utilizan.

Actividad 10. ¿Cómo nos organizamos en la comunidad?

Actividades del evaluador:

Pedir al alumno que investigue con sus familiares o algún vecino: ¿Existe alguna autoridad en la comunidad? ¿Qué nombre recibe? ¿Cuáles son sus funciones? ¿Qué reglas existen en la comunidad para organizarse y tener buenas relaciones? La actividad anterior se encarga como una tarea extraescolar que se pide que registre, y al siguiente día se cuestiona al alumno acerca de las respuestas que presenta, con el fin de obtener mayor información que la que fue capaz de escribir.

Actividad 11. ¿Cómo resolvemos los principales problemas en mi comunidad? Actividades del evaluador:

Comentar con el alumno acerca de los problemas que se presentan en la comunidad y qué actividades se llevan a 
cabo para darles solución. Pedir que se llene la siguiente tabla.

Actividad 12. El pasado de mi comunidad. Actividades del evaluador:

Solicitar al alumno que pregunte a personas mayores cómo era antes la comunidad. A partir de lo anterior indicarle que elabore dibujos (Nivel I), elabore un cuadro comparativo (Nivel II) o redacte un texto (Nivel III) en donde muestre los principales cambios identificados. Esta es una actividad extraclase.
Cada una de las actividades se incorporaron en dos cuadernillos. En el Cuadernillo 1, se presentaron las instrucciones para aplicaciones específicas de cada una de las actividades y las guías de registro para los investigadores, en el Cuadernillo 2 se ubicaron los formatos para recopilar las evidencias de los desempeños de los alumnos. A continuación se muestra un ejemplo de la presentación de una de las actividades, tal y como se incluyeron en los mencionados cuadernillos.

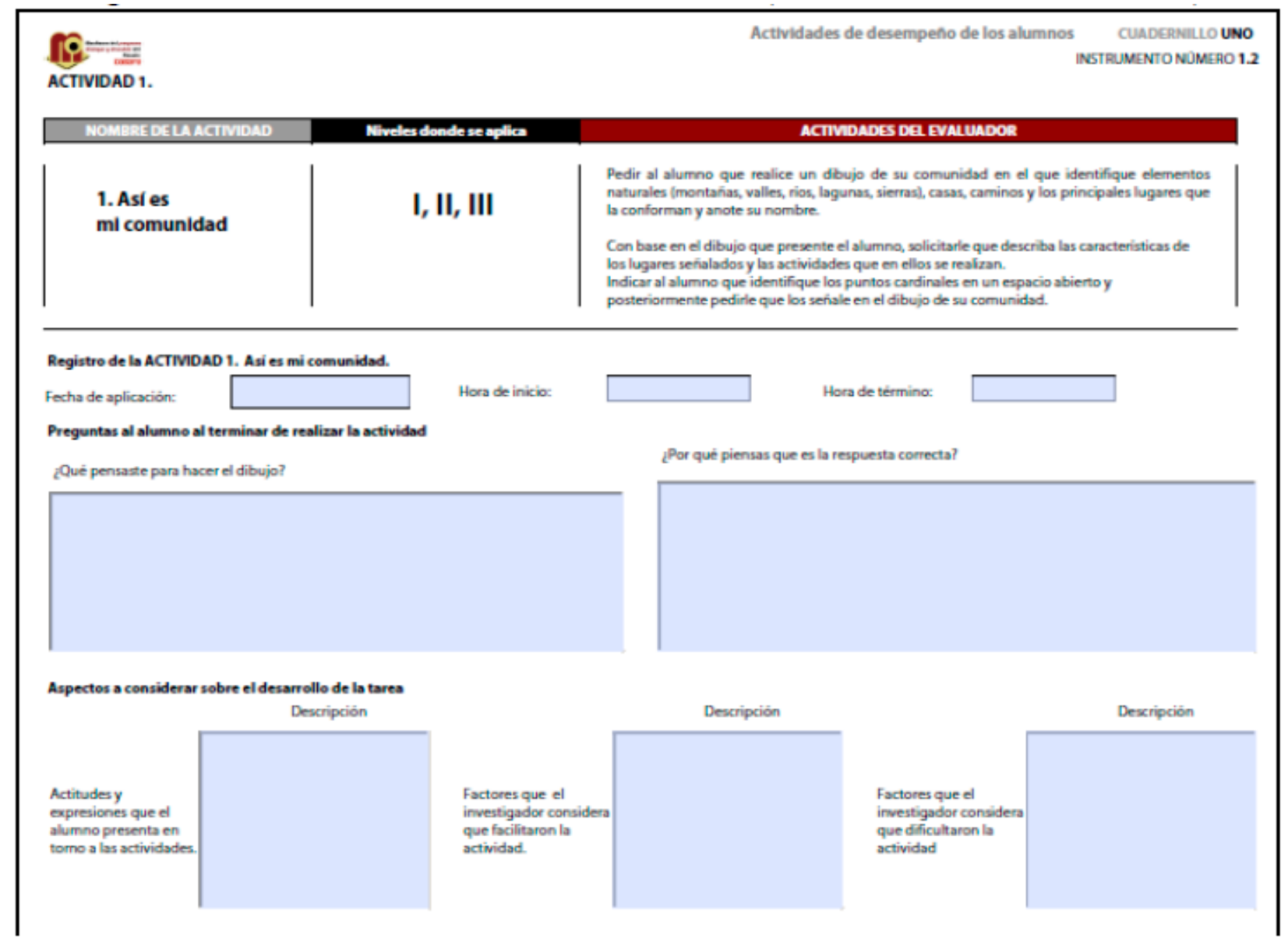

Figura 1. Actividad: Así es mi comunidad. (Tomado del Cuadernillo 1). 
RECIE. Revista Electrónica Científica de Investigación Educativa Vol. 1, núm. 2, enero-diciembre 2013, pp. 417-426.

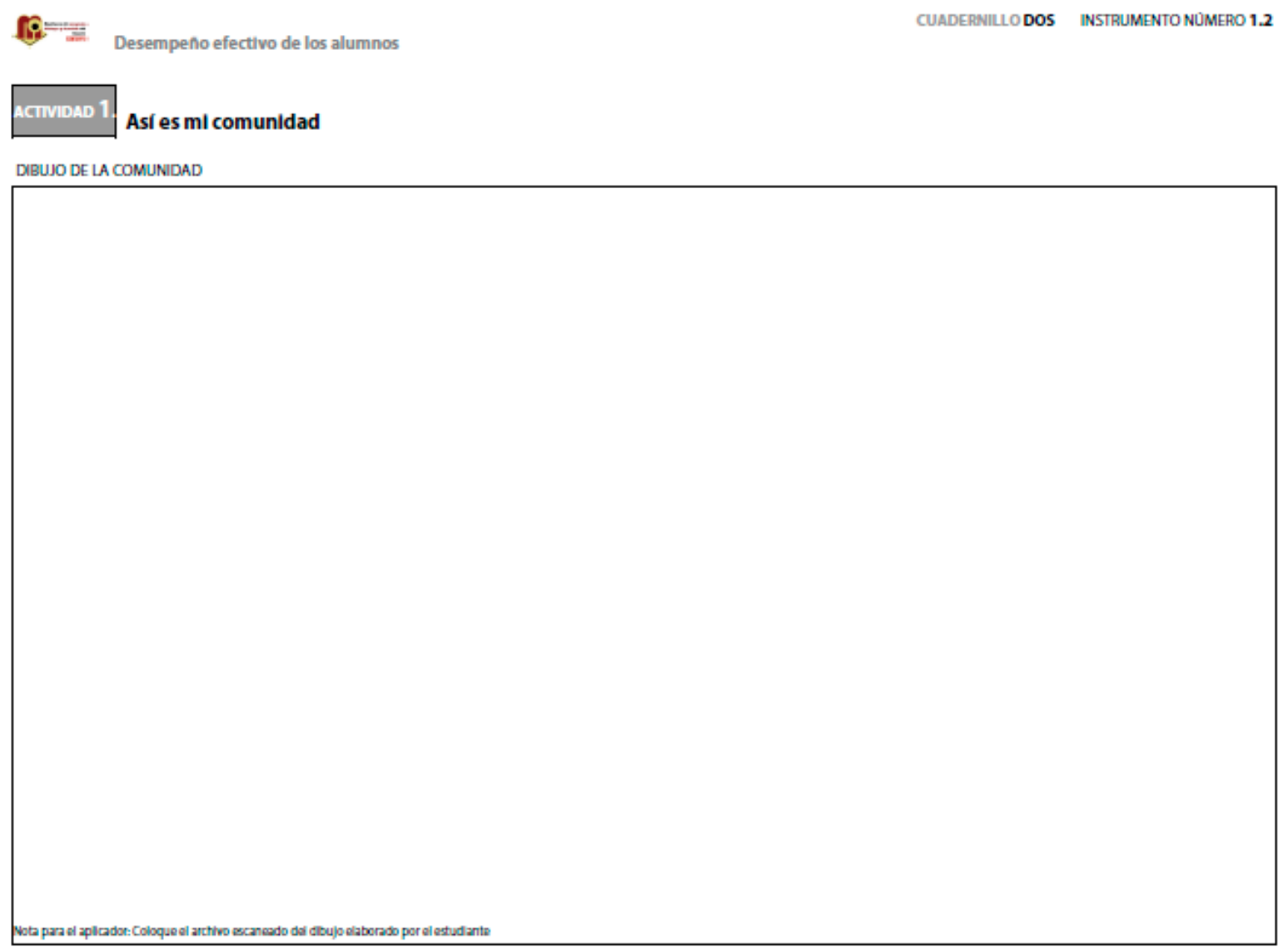

Hoja 1 de 11

Figura 2. Actividad: Así es mi comunidad. (Tomado del Cuadernillo 2).

\section{Definición de la muestra}

La aplicación del instrumento de desempeños efectivos se realizó con alumnos de aprovechamiento académico polarizado, inscritos en cada uno de los tres niveles de los 16 Centros Comunitarios en donde se realizó el monitoreo. (Ver Tabla 1). 
Tabla 1. Aplicación del instrumento de desempeños efectivos

\begin{tabular}{|c|c|c|c|c|c|c|c|}
\hline \multirow[t]{2}{*}{ Caso } & \multicolumn{2}{|c|}{ Nivel I } & \multicolumn{2}{|c|}{ Nivel II } & \multicolumn{2}{|c|}{ Nivel III } & \multirow{2}{*}{$\begin{array}{c}\text { No. de } \\
\text { alumnos }\end{array}$} \\
\hline & Alto & Bajo & Alto & Bajo & Alto & Bajo & \\
\hline Chiapas I & 1 & 1 & 0 & 1 & 0 & 1 & 4 \\
\hline Chiapas II & 1 & 1 & 1 & 0 & 1 & 1 & 5 \\
\hline Chiapas III & 1 & 0 & 1 & 1 & 1 & 1 & 5 \\
\hline Chihuahua & 1 & 1 & 1 & 1 & 1 & 1 & 6 \\
\hline Durango & 1 & 1 & 1 & 1 & 1 & 1 & 6 \\
\hline Guerrero I & 1 & 1 & 1 & 1 & 1 & 1 & 6 \\
\hline Guerrero II & 1 & 1 & 1 & 1 & 1 & 1 & 6 \\
\hline Jalisco & 1 & 1 & 1 & 1 & 1 & 1 & 6 \\
\hline Edo. México & 1 & 1 & 1 & 1 & 1 & 1 & 6 \\
\hline Michoacán & 1 & 1 & 1 & 1 & 1 & 1 & 6 \\
\hline Oaxaca & 1 & 0 & 1 & 1 & 1 & 0 & 4 \\
\hline Querétaro & 1 & 1 & 1 & 1 & 1 & 1 & 6 \\
\hline Sinaloa & 1 & 1 & 1 & 1 & 1 & 1 & 6 \\
\hline Tabasco & 1 & 1 & 1 & 1 & 1 & 1 & 6 \\
\hline Tamaulipas & 1 & 1 & 1 & 1 & 1 & 1 & 6 \\
\hline Veracruz & 1 & 1 & 1 & 1 & \multicolumn{2}{|c|}{1} & 5 \\
\hline \multirow[t]{2}{*}{ Total } & 16 & 14 & 15 & 15 & 14 & 14 & \multirow[b]{2}{*}{89} \\
\hline & \multicolumn{2}{|c|}{30} & \multicolumn{2}{|c|}{30} & \multicolumn{2}{|c|}{29} & \\
\hline
\end{tabular}

\section{Procedimiento de análisis}

Para analizar la información obtenida de cada una de las doce actividades planteadas, se procedió de manera inductiva, la información se concentró en bases de datos que permitieron identificar aspectos recurrentes relacionados con procedimientos seguidos por los alumnos y los resultados obtenidos, además de las declaraciones al ser entrevistados y las características de las actividades realizadas.

En la Figura 3 se presenta un ejemplo de los resultados parciales obtenidos de la aplicación de la Actividad
1. Así es mi comunidad, que presentó Soraida (Nivel 1), una alumna de un Centro Comunitario de Tamaulipas.

El análisis se basó en la teoría fundamentada o teoría emergente (Grounded Theory) que fue originalmente desarrollada por Glaser y Strauss. Este proceso de análisis facilitó la comprensión de los procesos de conceptualización de los alumnos, la identificación de patrones emergentes que surgen a partir de la información. 


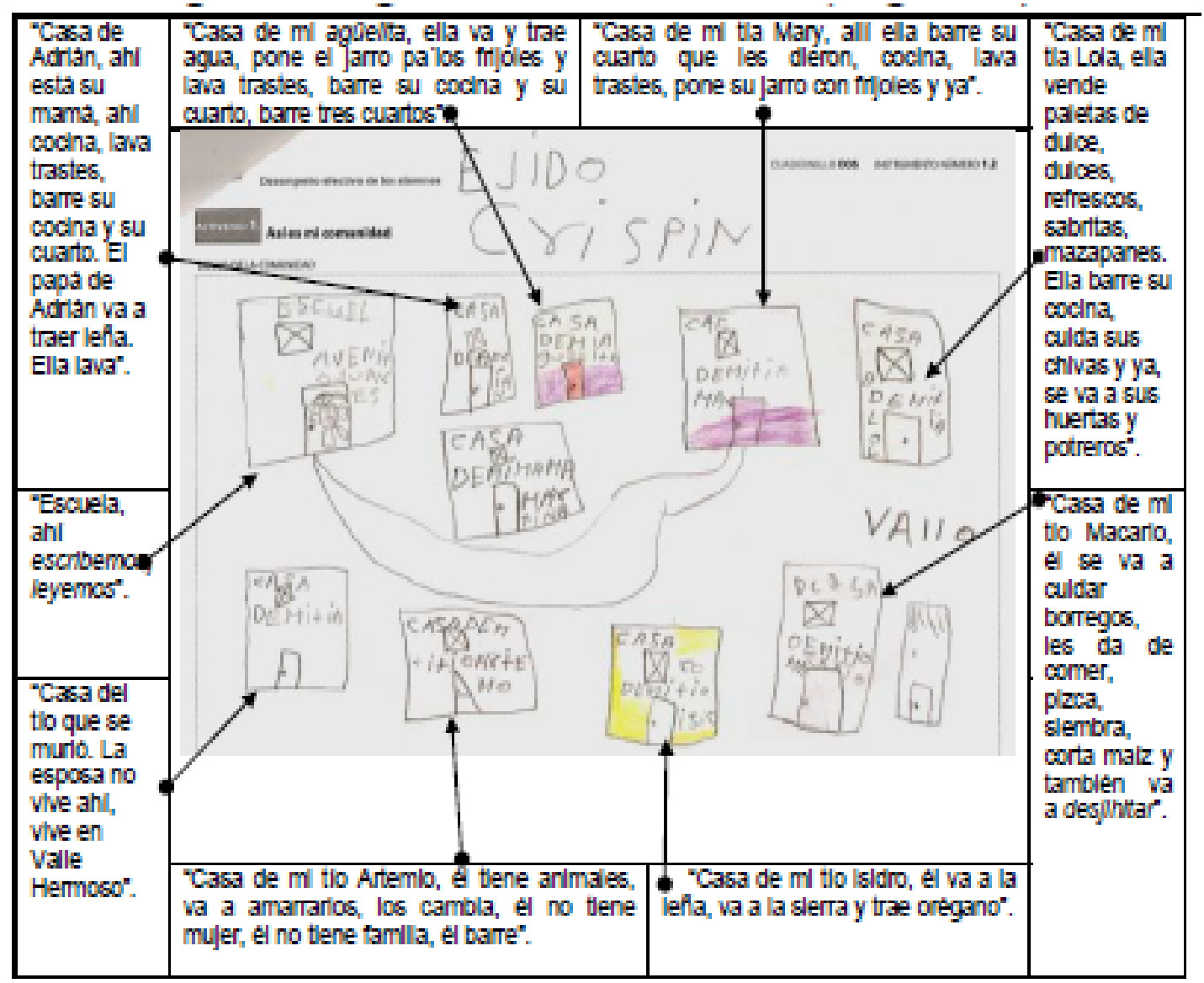

Figura 3. Registro de la Actividad 1 (fragmento).

A partir de los resultados del análisis de los desempeños de los alumnos, fue posible elaborar rúbricas en donde se identificaron diferentes niveles de ejecución para cada una de las actividades, para esto se consideraron las características de la aplicación y los resultados que realmente presentaron los niños, aunque inicialmente se habían estructurado rúbricas con base en los desempeños esperados de los alumnos, de acuerdo a lo establecido en el Plan y programas de estudio.

En la Figura 4, se muestra un ejemplo de la rúbrica correspondiente a la Actividad 1. 


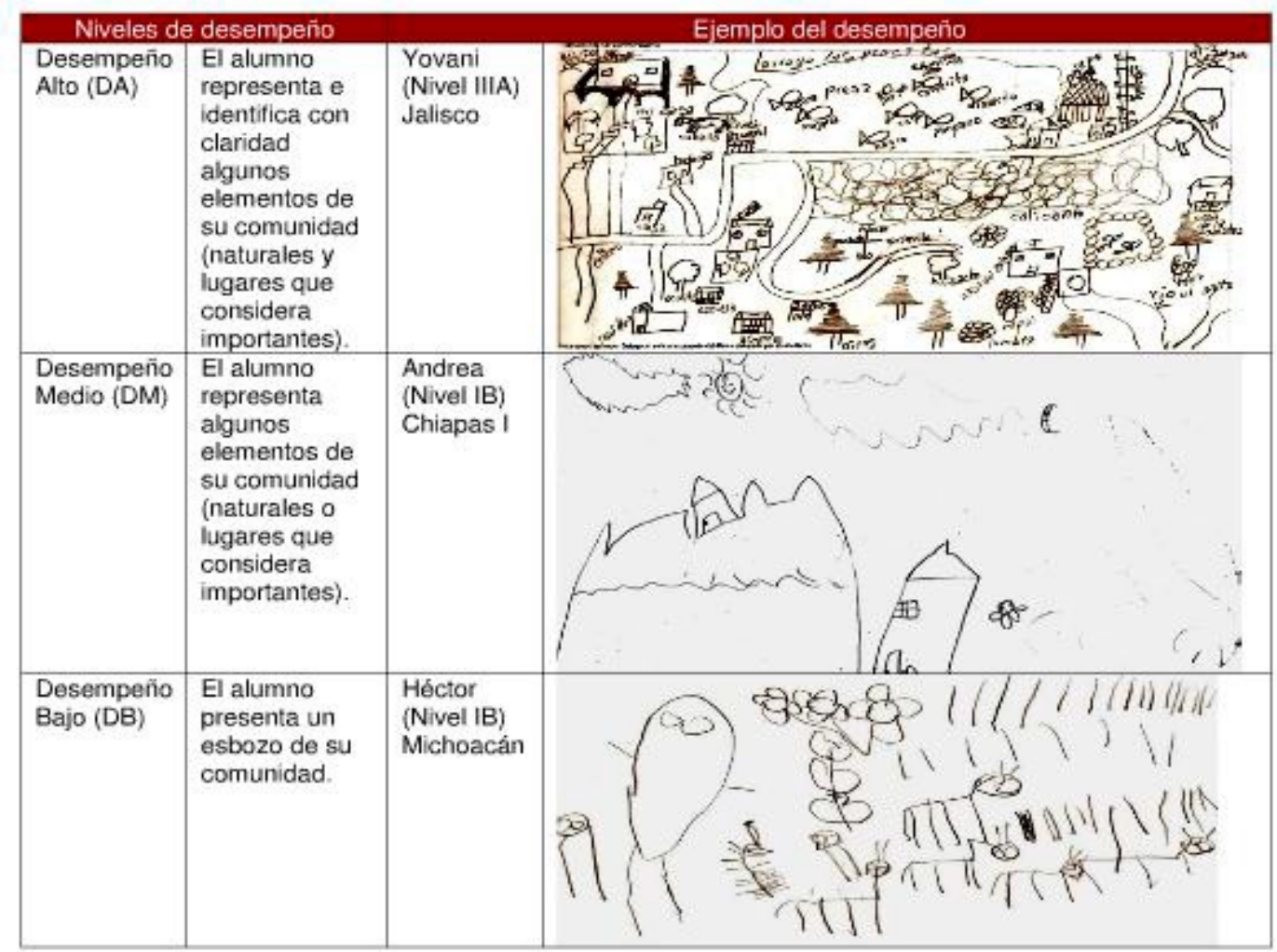

Figura 4. Rúbrica para valorar la Actividad 1

Los hallazgos derivados de la aplicación del instrumento de desempeños efectivos se agruparon en los siguientes temas:

Conocimiento del contexto inmediato; natural y social.

Características de la lectura.

Manejo de la lengua escrita.

Funcionalidad atribuida a las matemáticas.

El instrumento resultó funcional para conocer los desempeños de alumnos en aspectos curriculares básicos $y$ contribuir al análisis de formas de enseñanza que se realizan en Centros Comunitarios del CONAFE.

La elaboración de un reporte de los hallazgos permitió el logro de uno de los propósitos del Monitoreo de Dialogar y Descubrir, presentar a directivos de CONAFE, resultados de la aplicación de instrumentos sin ningún tipo de interpretación, para detonar reflexiones y conversaciones en torno a la situación que desde este estudio se percibían en relación a los aprendizajes observados en los alumnos. Sin embargo la información derivada del análisis de los resultados arrojados, de acuerdo con Strauss y Corbin (2004) puede también revisarse al considerar fundamentos teóricos, estos autores plantean en esta actividad las siguientes ventajas: Los conceptos derivados de la literatura pueden proporcionar elementos para realizar comparaciones entre los datos que emergieron del estudio. Establecer semejanzas, coincidencias y divergencias con los de la literatura. La revisión de la literatura puede permitir analizar alguna información que en apariencia puede parecer intrascendente y cobrar significancia. Incluso propiciar que emerjan nuevas categorías e información adicional a las que del estudio surgieron, 
estimula preguntas al identificar posibles discrepancias entonces, se extienden validan y refinan los conocimientos que se presentan. En síntesis, la literatura sobre los hallazgos se puede utilizar como una herramienta analítica que contribuya a la mejora de la conceptualización lograda.

\section{Limitaciones del instrumento}

La información obtenida requiere triangularse con datos derivados de otras técnicas procedimientos e instrumentos, para ampliar la posibilidad de comprensión de las prácticas pedagógicas.

Las principales limitaciones que los investigadores reportaron en referencia al instrumento, tienen relación con el tiempo requerido para su aplicación. Las actividades se desarrollaron por los alumnos de manera individual y se establecía respetar sus ritmos de trabajo, estas situaciones afectaba la programación para aplicar otros instrumentos durante la semana destinada para el trabajo de campo.

Resulta necesario contar con la totalidad de actividades resueltas por los alumnos seleccionados y que cada una reporte un mínimo de información. Esto no siempre fue posible, en el Caso Chiapas II, no se presentaron suficientes evidencias para valorar las actividades de Adrián (Nivel IIB) y Lisbeth (Nivel IIA) se enfermó y no concluyó la aplicación. En dos casos Chiapas III María (Nivel IB) las actividades desarrolladas por la alumna fueron mínimas, la alumna habla poco español, su lengua es el Chuj, al igual que en el Caso Oaxaca donde se suspendió la aplicación de actividades con Karina (Nivel IB) e Irma (Nivel IIIB) debido a que ambas niñas hablan en Chinanteco.

\section{Aprendizajes derivados}

En el ámbito educativo se hace necesario diseñar instrumentos que permitan ampliar las posibilidades de información y develar los resultados de prácticas pedagógicas en contextos específicos, además de documentar las experiencias obtenidas de su aplicación.

La identificación de desempeños efectivos de los alumnos a partir de un formato de actividades cotidianas permitió observar como los alumnos hacen uso de sus conocimientos y habilidades. Esta estrategia supera las limitaciones que presentan los denominados exámenes de lápiz y papel.

\section{Referencias}

Consejo Nacional de Fomento Educativo (2006). Manual del Instructor, Nivel I y II. México, D.F.: Editora Xalco.

Consejo Nacional de Fomento Educativo (2006). Manual del Instructor, Nivel III. México, D.F.: Editora Xalco. Shagoury, R., Miller B. (2000). El arte de la indagación en el aula. Manual para docentes-investigadores. Madrid: Gedisa editorial.

Strauss, A., Corbin, J. (2004). Bases de la investigación cualitativa. Técnicas y procedimientos para desarrollar la teoría fundamentada. Bogotá: Editorial Universidad de Antioquia.

\section{Agradecimiento}

Al Dr. Armando Loera Varela, director de Heurística Educativa e investigador principal del Monitoreo Dialogar $y$ Descubrir, por las sugerencias $y$ comentarios planteados para las actividades que conformaron el instrumento. 